\title{
The World Physical Triad: Matter, Antimatter and "Dark Energy" in the Processes of Climatic Changes on the Earth
}

\author{
Robert A. Sizov* \\ Solid State Physics Institute, Chernogolovka, Russia \\ Email: sizov.robert@gmail.com
}

Received 5 February 2016; accepted 27 March 2016; published 31 March 2016

Copyright @ 2016 by author and Scientific Research Publishing Inc.

This work is licensed under the Creative Commons Attribution International License (CC BY). http://creativecommons.org/licenses/by/4.0/

(c) () 0 pen Access

\section{Abstract}

The opening and many years of research of magnetic spinor particles (real magnetic charges) in atoms and substance have enabled the author to formulate the conception of the Physical Triad, according which the real World consists of three fundamental phases: Matter, Antimatter and Energo-phase (Energo-medium). Particles of Matter are called spinors and particles of Antimatter are called antispinors. Energo-medium is a gasiform phase of high density that fills by himself all the infinite space of the real World. It consists of spinless and massless particles-energions. Spinor fields can be both flows energions (fields of Matter), so and anti-flow energions (fields of Antimatter). Atomic-shaped structures consisting of electric and magnetic spinor particles represent a Physical Mass (atoms, nucleons, etc.). The main characteristic of all varieties of Mass is its ability radiate gravitational field, which is a vortex electromagnetic field. All spinor particles are massless so as individually generate a gravitational field they can't. All primary forces in the real World are implemented by means of Energo-medium, i.e. contact pressure its particles-energions. The spinor fields, including the gravitational field, myself the real of the power significance, have not. They are only intermediaries, inducing in Energo-medium its active (power) education, which is called "Dark Energy". "Dark Energy" can be both positive, so and negative. Namely, a positive "Dark Energy", which is associated with the technical activity of man, is responsible for stable climatic changes on Earth. Greenhouse gases are not the main "culprit" of climatic changes on our planet. However, these gases are the simplest indicator of the overall level of irreversible physical processes that stimulate the growth of the positive "Dark Energy" and are responsible for the negative thermal scenario on Earth.

\section{Keywords}

Magnetic and Electric Spinorial Particles (Spinors and Antispinors), Antielectrons, Magnetons,

*At present works as individual researcher, Dr. of physical and mathematical sciences. 
Antimagnetons, Bispinor, Physical Mass, Vortex Electromagnetic (Gravitational) Field, Matter, Antimatter, Energo-Medium, Energions, "Dark Energy”, Climatic Changes

\section{Introduction to the Physics of Electric and Magnetic Spinor Particles and the Physical Triad of the Real World}

The magnetic spinor particles (magnetic charges), which are the immediate sources of all magnetic fields in Nature, have been experimentally found by the author in the structures of atoms and substances and first introduced to the scientific community in 2001 in the publication [1]. It should be noted that the initial experiments, which prompted the author to studies the problem of participation of magnetic spinor particles in the structures substance were his experiments with magnetic scattering of neutrons in ferrimagnetic crystals [2] [3]. The results of the main experiments of the author, which related to the detection and studies of real magnetic charges and their currents in the atoms and the substance, are given in [4], [5] and in addition, their brief annotation is given in [6].

Magnetic spinors in compositions of the atomic shells (author's name-magnetons) are fundamental particles of Matter, which by their physical parameters are the magnetic analogs of electrons, i.e. have a magnitude of charges and spins, equal to the corresponding values for electrons. Magnetons like electrons have a charge with a negative sign $\left(\mathrm{g}^{-}\right)$. They belong to the class of leptons and along his statistical properties to fermions (spin 1/2).

Magnetic antispinors, for example, antimagnetons, are true antiparticles to magnetons. They have a positive charge $\left(\mathrm{g}^{+}\right)$and belong to the class of antileptons. According to statistical properties, antimagnetons are antifermions with antispin $(-1 / 2)$ on relation to spinors.

Note 1. It should be added that the mere detection of magnetic spinor particles (magnetic charges), which managed to make the author of this article, should not be seen as something unique. Merit of the author was only in the fact that he showed real participation of the magnetic charges in the structures of atoms, substance and, in general, in the compositions Physical Mass (PM). Of course, this is a very important conclusion. This was followed by a chain of physical findings and experiments that, in the end, and led the author to his Gravito-Physics and Gravito-Technics [7]-[9]. If we talk about the first real detection of magnetic charges, it is the great merit of Felix Ehrenhaft, who made their discoveries in the early 20th century, and which were repeated by many of his followers [10], [11]. The reasons that more than a hundred years blocked recognition of fundamental experiments and valid conclusions F. Ehrenhaft were discussed in detail in the publications of the author [4]-[6] and noted in this article..

The most famous representative of electric spinors is electron. According to the researches of the author, the true antiparticle for electron, in fact, is the real antimaterial particle with a charge of $\mathrm{e}^{+}$, which is neither the positron nor the Dirac's "hole" [12]. Fortunately, unlike from real magnetic charges, the presence of the positive electric charges in substance is not a surprise for modern physical science. The merit of the author in solving the problem with the real status of the positive charges in the atoms and substance is possible to consider so-called "antimaterialization" Dirac's "holes". In addition, the author shows why the observed universally positive electric charges have not been entered in the category of real fundamental particles (antispinor), and almost a hundred years, in accordance with the erroneous point of view, Dirac's, have been recognized in modern physical theory as quasi-particles, so-called "holes" or vacancies of electrons. The main physical parameters of the magnetic and electric spinor particles (magnetons, antimagnetons and antielectrons) constituting together with electrons of atomic shells, are contained in Appendix 1.

Representatives of weak spinor particles, i.e. the particles involved in weak interactions are neutrinos and antineutrinos, which are discussed in detail in the publications of the author [13]. It should be noted that the detection by author of magnetic spinor particles as structural components of atoms and substance allowed him to assume that in addition to the electro-weak particles in the World can exist and magneto-weak particles, for example, the magnetic neutrinos, that emit magneto-weak fields. Depending on the speed of propagation of spinor fields in space, their sources, i.e. spinors and antispinors, can be divided into two groups (block): particles electromagnetic block and block weak particles. The speed of propagation fields, emitted by the particles of electromagnetic block, i.e. by electric and magnetic charges, is equal to the speed of light. The speed of propagation of weak spinor fields is always higher than the speed of light, but, most likely, only in times. 
In addition, the intensity of weak fields significantly weaker than the fields emitted by the particles of the electromagnetic block, what determined the name "weak" particles. More information about the speed characteristics of the spinor fields and spinor particles is given below (see Section 3).

The results of years of research real magnetic spinor particles allowed the author to formulate the conception of the World Physical Triad, according to which the real World consists of three fundamental phases: Matter, Antimatter and Energo-medium [13]. Phases Triad self-sufficient and their conservation laws forbid any mutual transformations between particles of different phases. For example, the law of conservation of Matter eliminates the existence of any pre-matter particles. Material particles can only turn in the Matter particles with more or less significant content Matter in them. All material particles are spinors and antimaterial particles-antispinors. Spinors and antispinors referred to as the General title: spinor particles. The particles Antimatter compose approximately half of all real spinorial, i.e. charged particles in the World and their absence in the physical representations is determined by the Physics of their retention in substance or conditions of their confinement. The law of conservation of Antimatter eliminates any possibility for transformation antispinors in particles of other phases of the Physical Triad, answering the law of conservation of Antimatter in the real World. The process of pair annihilation: spinor-antispinor, is accompanied by a strong compression (pressing) of these particles to one another by forces "Dark Energy" and, in principle, not lead to the humiliation of the particles. In such process are only possible transformations, for example, antispinors in other antispinors with great or less quantities of Antimatter in them.

Energo-phase (Energo-medium, Power-medium, En-medium) is a global the world phase, which executes all the primary acts of force action on particles and mass in the real World. Energo-medium is a gasiform phase of high density that fills by himself all the infinite space of the real World. It consists of spinless and massless particle-energions, which by their Nature have no relation to the Matter or Antimatter. Energions are very small, they move in all directions at speeds close to the speed of light and can only be of two types: the Left $\left(\varepsilon_{\mathrm{L}}\right)$ and Right $\left(\varepsilon_{\mathrm{R}}\right)$ energions, that should be linked with the appropriate direction of their own rotations. General state of energions within the Energo-medium is determined as doubly degenerate. Super high mobility of energions allows the particles and masses to move relatively freely in the Energo-medium when this medium is in basic (undisturbed) state. According to very rough estimates of the size energions $\mathrm{d}_{\varepsilon}<10^{-35} \mathrm{~cm}$ and density Energo-medium $\rho_{\varepsilon}>10^{90}$ energions in $\mathrm{cm}^{3}$. The latter values are given in accordance with the data, which offer for particles and density medium of traditional Ether [14].

Note 2. It can assume that if energions, i.e. particles of Energo-phase, stop moving, all spinor particles and Masses, including planets and stars, will be "concreted" in a static world environment. Such a scenario can be defined by the term of "exotic death of the Universe". Fortunately, this can't be, because not can be never.

According to the concept of Physical Triads, primary forces which operates in the real World, implemented by Energo-medium through the contact pressure of its particles energions on the spinor particles, and therefore on masses (bodies). Forces direct action on the particles and masses, which are implemented in the Energophase, called the forces of "Dark Energy". They represent the fields of nonequilibrium states in Energo-medium in the form of zones of local pressure created by the particles-energions. The formation of "Dark Energy" in the Energo-medium is carried out by means of spinor fields, i.e. fields of charged particles. In so doing all variety of spinor fields, including the gravitational field does not have a significant of force value. They only play the role of intermediaries exerting influence on energo-state of the Energo-medium and inducing formation of "Dark Energy" in it. Namely "Dark Energy" is real power factor, performing the whole dynamics of particles and bodies, as the scale of the Universe (the movement of galaxies, stars, planets and other objects) so and in the microcosm (for example, the dynamics of spinor particles within a physical masses, such as in atoms and nucleons).

As a historical prototype of the Energo-medium and its particles-energions, the author looks at the environment “ the world particles”, suggested by Le Sage, G. L. (1784) [15]. These particles, according to the existing descriptions, are particles of unknown nature, which are very small, are moved in all directions with high velocities and are poorly absorbed by the substance. Between two bodies, by according to Le Sage, there is an effect of shielding the flows of "the world particles", which creates conditions for the pressing of bodies to each other under the action of forces, which and represent the gravitational forces. In the author's publications [13] it is shown that the pressing of bodies in the framework of gravitational interactions is true, however, for reasons that are different from the interpretations by Le Sage. By structural form and some signs of the Energo-medium reminds to long-suffering the Ether, whose particles are not bound to Matter. However, they are the original "building materials" for all kinds of material particles and their motion is the basis of the force fields. However, 
between Energo-medium and Ether are a number of fundamental differences. So, particles of the Energo-medium is not reducible neither to Matter nor Antimatter, because Energo-medium is a self-contained the world phase, which implemented in the framework of the law of own self-preservation. Dynamics of particles in Energo-medium characterized by both linear so and angular moments, while the particles of the Ether are attributed to only one form of movement: forward motion. The latter circumstance determines substantial differences in the forms and properties of fields generated by charged, i.e. spinor particles, in the Energo-medium and in the medium of traditional Ether.

Significantly different Energo-medium and from the Physical vacuum, which is widely used in modern theoretical approaches. Latter is often represented as the lowest state of quantized fields, bereft of any real particles. In the absence of real particles, there is no real pulses, i.e. disappears the concept of "energy", as in relation the medium so and fields of the Physical vacuum.

It should be noted one more moment. So as Energo-medium is the carrier of world energetic, its fundamental property must be reflected inevitably in its name.

Figure 1 shows a diagram of the Physical Triad of the real World, which consists of three fundamental phases: Matter, Antimatter and Energy medium. Spinors, antispinors and energions are shown in the diagram particles of Triad. Noted on Figure 1, such derivative from phases Triads is as the spinor fields and "Dark energy".

Outside of this scheme remained the condensates, consisting of both spinor particles, so and so-called the energion condensates, consisting of spinor fields. The condensates, consisting of a spinor particle, can be divided into two classical groups: Gravito-Forming structures (GFS) or the Physical masses and Gravito-notforming mediums (GnFM). The most striking example of gravito-not-forming mediums, according to the author, can serve as Black holes. Example pure energionic condensates, in compositions of which there are only spinor fields are photons. Details concept of the Physical Triad real World contains in the two editions of the author's book [13].

\section{Physics Confinement of Magnetic Charges and All Antispinors in the Substance Is Main Reason Their Disregard by the Physical Science}

As noted above, the main reason that the real magnetic spinor particles constituting about half of all real spinor particles in Nature, as well as electric antispinors, i.e. the true antielectrons, were not recognized in physical science, determined by the Physics of their retention (confinement) in the atoms and the substance that is fundamentally different, for example, from the confinement of electrons. Hypothesis confinement of fundamental particles was put forward to explain the retention of quarks inside hadrons, and also to explain the negative results in the search for free quarks. The notion of confinement, with much more base than for quarks, needs to be expanded on hold magnetic charges and the real antielectrons in atoms and substance, and also for explaining

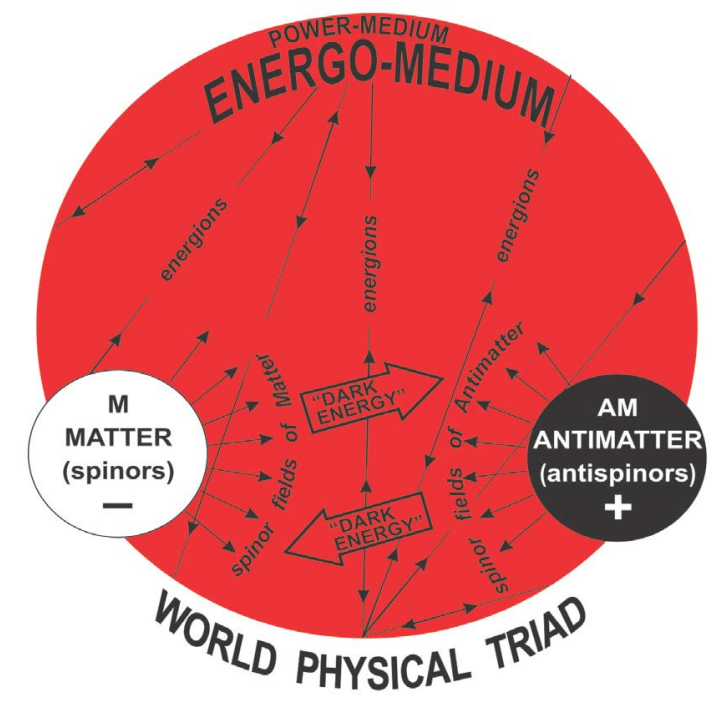

Figure 1. The physical triad of the real world. 
the ban on their free exit from substance. It should be noted that the conditions of confinement of the magnetic spinor particles and of the true antielectrons in the substance substantially softer than conditions confinement of quarks in hadrons. On the other hand, the conditions hold (confinement) antielectrons and magnetic charges in substance are substantially more rigid in comparison, for example, with confinement of electrons.

Figure 2 shows the layout of levels of electrons, magnetic charges and antielectrons in the condensed state of substance on the scale of energy. It is well known that the electronic levels are located in a potential pit and for going out of electron in a free state is necessary to increase the internal energy of a substance, for example, by heating.

Unlike electrons, the energy levels of antielectrons, as and the levels of magnetic charges, are under potential cupola and for the implementation of the free state these particles substance need cooled, that is, to reduce its internal energy. The deep stages of cooling there arises the superconductivity effect what probably is an evidence of transition of the magnetic spinor particles, as and antielectrons, into the condition free from bonds with the lattice of superconductor. In this case, the electric current passes through the superconductor without resistance, since the free magnetic charges, which revolve around the current lines, do not experience friction the lattice about the superconductor. With increasing internal energy the substance (by heat, radiation, etc.) realized the transition of magnetic charges on higher energy levels, what is always accompanied by a sharp increase in the electrical resistance of the conductor [5], [6], [12].

Thus, under "sanctions" confinement, i.e. under the cupola (see Figure 2) there were three real spinor particles of the four components of the atomic shells, i.e. a pair of magnetic particles with charges $\mathrm{g}^{-}$and $\mathrm{g}^{+}$and antielectron with a charge of $\mathrm{e}^{+}$. Recall that the value of the particle charge in the atomic shells satisfies the condition: e = g. Paradoxically, these three real spinor particles are still not recognized by modern physical science. Real magnetic charges were "compensated" by means of such theoretical prosthesis as magnetic moments. Theoretical "fate" of the true-antielectrons, i.e. the real particles of Antimatter was "solved" by P. Dirac, who "buried" this particle under the erroneous concept electronic vacancies or "holes". Is it any wonder after all this, the incredibly complex state of modern physical theory?

As shown in the publications of the author [1]-[9] and also in this article (see, Section 4), shells of the atoms, in reality, are not electronic but are composed of electric and magnetic spinor particles. Joint orbital currents of electric and magnetic charges within atomic shells are the sources of gravitational field which is a vortex electromagnetic field. Thus, the timely introduction of magnetic charges in the atomic shells, would avoid numerous and grandiose delusions associated with gravitational manifestations.

\section{Spinor Fields of Matter and Antimatter [13]}

The spinor fields, which are generate by spinor particles, depending on nature of particles are divided on fields of Matter and Antimatter. As united name of these fields in present article, the term "the spinor fields" is used, which is synonym with the term "the fields of charges". Fields of Matter represent himself flows of energions which is polarized by their linear and angular pulses. Sources of fields Matter are all material particles, which called spinors. Therefore, fields Matter you can define and as fields of the spinors. Terms: "spinors", "antispinor" and "spinor

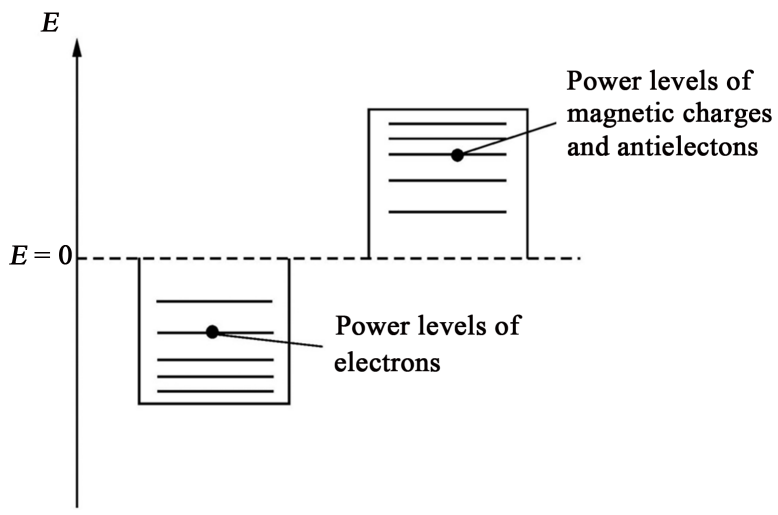

Figure 2. Location on the scale of the energy levels of electrons (in the potential "pit") and magnetic charges with antielectrons (under potential "cupola”) in substance. 
fields" are derived from such thing as spin of the particles which determined by their rotational dynamics. Spin (angl.) - the rotation. The spin is represented as vortex vector, the value of which is determined by the angular velocity of proper rotation of the spinor particle. Another important parameter of the dynamics of spinor particles, is direction of their rotation. This parameter is not directly associated with spin, but he is determiner the very important essence of the particles of Matter and Antimatter. Spinor particles interact with particles Energo-phase, with education flows or antiflows energions which and are real spinor fields. Spinors, i.e. particles of Matter, in such processes perform the role of a natural time-of-flight selector, rotational dynamics and natural type of which determine the parameters of energions in the flows fields, i.e. their linear pulses and rotational type (Left- $\varepsilon_{\mathrm{L}}$ or Rait- $\varepsilon_{\mathrm{R}}$ ). Depending on the magnitude (module) of the spin vectors, i.e. depending on the angular velocity of rotation of the spinors, fields of Matter can be divided into two oblasts: electromagnetic (EM) and weak. Fields EMoblastare such well-known fields, as electric and magnetic. The spinors, i.e. the sources of fields Matter of EMoblast are, for example, electrons and magnetons, which together with the relevant antispinors make up of electromagnetic shells atoms. Spinors of the EM-oblast include and such particles as minielectron and minimagneton, which can are components of EM-shells nucleons.

Consider the speed characteristics of energions in various spinor fields. Figure 3 shows a probabilistic spectrum of energions on their linear velocities in the main state of Energo-medium. The line on the spectrum designated as I, correspond to the speed of light (the condition: $\mathrm{u}_{\varepsilon}=\mathrm{c}$ ) and defines group energions that form the fields of the electromagnetic oblast. These are electric and magnetic fields, which, as known, are distributed in space at the speed of light. $\mathrm{N}_{\varepsilon}$ - the number of energions in the compositions of spinor fields

Energions, its own linear velocity which are located on the spectrum to the right of line $\mathbf{I}$, form a weak spinor fields. The spinors, which emitting a weak fields are, for example, the material neutrinos, which can still be called a weak spinors. Weak spinors are characterized by greater magnitude of the spin vector ISI than EM-spinors, what corresponds to greater angular speed their own rotation. Thus, the velocity of weak fields in Energo-medium (space) exceeds the speed of light, but most likely at times. Since the propagation velocity of a spinor field is the maximum possible speed of the translational motion of its source, linear speed, for example, neutrinos can exceed the speed of light. As already noted, weak spinors are very small in size, which, for example, for neutrino evaluated as $10^{-21}-10^{-23} \mathrm{~cm}$. However, despite its small size and weak (in intensity) of the fields, the weak spinors, such as neutrinos, is able to initiate the destruction of such giants of the world of elementary particles, as neutrons.

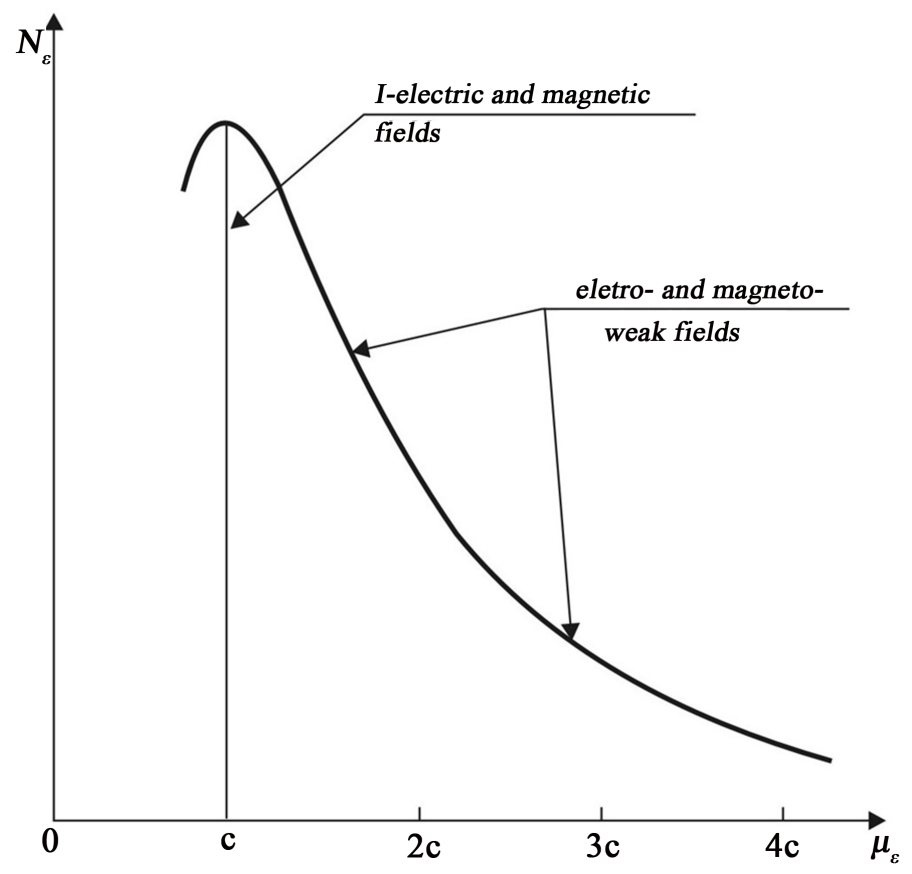

Figure 3. The estimated spectrum of energions on their linear velocities in the ground state Energo-medium. 
Analyzing the sources of fields Matter you should pay attention to the fact that depending from the nature of their interaction with energions, they are divided into two groups: electro-spinors, fields which contain the Left energions $\left(\varepsilon_{\mathrm{L}}\right)$ and magneto-spinors which contain the Right energions $\left(\varepsilon_{\mathrm{R}}\right)$. Flows left and right energions propagating in a Energo-medium at the speed of light, are respectively well-known electric and magnetic fields.

In their publications [13], the author is emphasized that in the interactions of energions and the spinor particles, separately, are implemented put into practice of the laws conservation of both linear and angular impulses of the particles involved in these interactions. Therefore, if the linear pulses energions are responsible for the linear dynamics of spinor particles, then the angular pulses are responsible for the rotational dynamics of the spinors and antispinors in Nature.

Consider further the fields of Antimatter, which are forming the antispinors i.e., particles of Antimatter. Recall that the spinors, i.e. particles of Matter, emit flows of polarized energions that and represent the fields of Matter. The processes of formation (birth) of flows energions in Physical science are associated with a negative charge source of fields, i.e. with spinors.

Fields of Antimatter are antifields in relation to the fields of Matter and manifest in processes of depolarization or annihilation of flows energions, which the spinors is emitted in the form of fields of Matter. Thus, if the spinors are poles birth of flows polarized energions, antispinor are antipoles, i.e. drain or destroyer such flows. The fields Antimatter can be defined as antifields polarized energions if correlate with the fields of Matter.

Mutual status of the fields of Matter and Antimatter is convenient to consider the example of the electric bispinor or of the spinor electric dipole, depicted on Figure 4. Here the spinor (electron), in the role of time-of-flight selector "cuts out" from the spectrum of particles Energo-medium the flow of energions, which are polarized along their linear and angular impulses. This flow and is the spinor field, in this case by electric field. The maximum flux density energions $\rho_{\varepsilon}\left(\mathrm{N}_{\varepsilon}\right)$ or tensity the spinor field emitted by the spinor, will be at the exit from the spinor and equal to zero at the entrance in antispinor.

Such a fields is determined by the negative density gradient polarized energions in the flows fields spinor, i.e. $\frac{\mathrm{d} \rho}{\mathrm{d} r} \cdot \boldsymbol{r}_{0}<0$, where $\boldsymbol{r}_{0}$ is the unit vector in the direction of the vector spin the spinor $\boldsymbol{S}_{\mathrm{s} p}$. Because antispinors "engaged" the depolarization (destruction) of flows of polarized energions, i.e. they perform the role of antiselector such flows, the maximum density of antiflow is observed at the output from antispinor, and minimum at the inlet to the spinor. Field of antispinor is determined by a positive density gradient polarized energions in the flow and has form $\frac{\mathrm{d} \rho}{\mathrm{d} r} \boldsymbol{r}^{\prime}>0$, where $\boldsymbol{r}^{\prime}$ is the unit vector in the direction of the spin vector $\boldsymbol{S}_{a s}$ antispinor. The ratio of density flows and anti-flows of the energions, i.e. relation tension of the fields of Matter and Antimatter for the case of electric bispinor is shown on Figure 4.

In connection with the above description of the fields of antispinors, it should be noted that these fields were left "overboard" serious scientific attention. If the fields of Matter, such as electric fields, is well known in science and technology, fields of Antimatter in modern physical theory are missing or, if present, then implicitly. However, about the effects of mutual repulsion of positively charged particles (charges) informed even the schoolchildren and the fact of such repulsion is a definite sign of the existence of fields positive charges, i.e. fields of Antimatter.

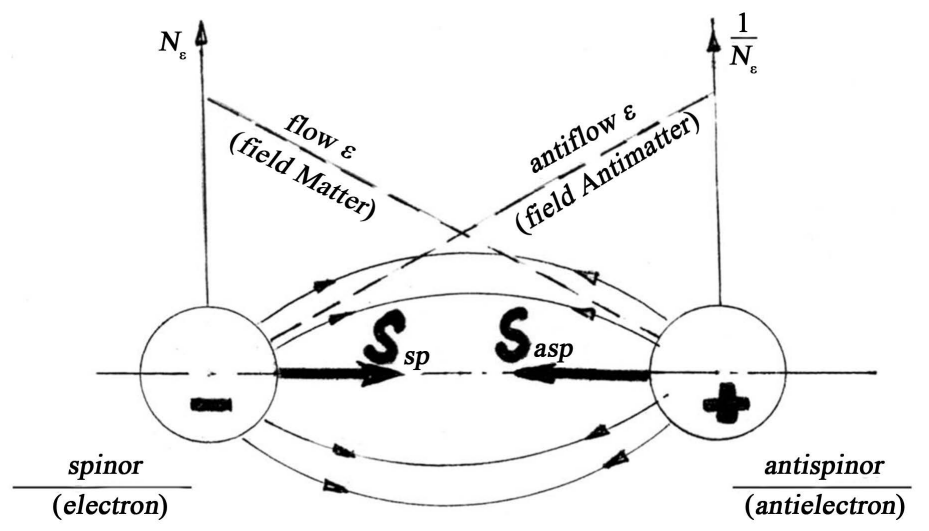

Figure 4. The electric dipole (bispinor) as source of fields of Matter and Antimatter. 
It is important to note that the manifestation of fields Antimatter is not limited to their influence on the fields of Matter. In the absence of spinors and their fields, antispinors depolarize those energions in composition Energomedium, which could form the appropriate fields of Matter.

Introduction to physical concepts such thing as fields of Antimatter or anti-fields, allows to extend the concept of parity, which are applied to the spinor particles and on to the spinor fields. If assign for fields of Matter natural minus sign and for fields Antimatter no less natural a plus sign, properties of functions these fields will meet the conditions, as spatial inversions, so and inversion their sign.

\section{The Essence Real of the Physical Mass. Vortical: The Magnetic and Electromagnetic (Gravitational) Fields and Gravito-Levitation}

Since 2001 ([1]), the author in all his messages tried to explain that Physical Mass, for example, atoms or nucleons, is the electromagnetic spinor structures, consisting of four independent fundamental particles: two spinors (electric and magnetic) and two antispinors, corresponding to them. Without this quartet of Physical Mass cannot be formed. Main characteristic feature of all varieties of Physical Mass is their ability to the emission gravitational field, which is vortex electromagnetic on his nature. It is important to note that namely "thanks" the Physics retentoin (confinement) of spinor particles in atoms and substance, as magnetic spinor particles so and electric antispinors, for example, the true antielectrons has been "buried alive", and, in fact, been closed any reasonable approaches physical theory to real the essence of Physical Mass. The latter circumstance, according to the author, determined the occurrence of the famous "divine" of the Higgs boson which is the quantum of the Higgs field. The very same Higgs field is fictitious, exclusively by the mathematical "donor" Mass in Nature. All spinor particles as spinors so and antispinors are massless, since the Mass is the result of their joint participation in forming the atomic-shaped structures. It is also important to emphasize that massless are not only electric and magnetic spinor particles, for example, electron and magneton, but also weak spinor particles, i.e. neutrinos of all types. To ascribe of the spinor particle mass is absolutely absurd, because they have the ability to emit only their characteristic spinor fields: electric or magnetic (electromagnetic block spinor particles), or the electroweak and magnetoweak (block weak spinor particles), (see [1] [5] [12]). A Mass can still be called as Gravito-forming structure, which is formed by electric and magnetic spinor particles, i.e. by particles of electromagnetic unit. Since the gravitational field is the electromagnetic field, it is not difficult to understand that it can propagate in space only at the speed of light.

It is important to emphasize that the Mass and, for example, Matter of completely different physical categories. Matter, as fundamental phase of the World Physical Triad, includes all material spinor particles (spinors), as the electromagnetic block, so and block the weak particles. Since all varieties of Mass are composed of material and antimaterial spinor particle, to ascribe Mass of the Matter particles is incorrect by definition. So, if, for example, to called protons and neutrons, as particles of Matter-that is completely incorrect, because the nucleons are varieties of the Mass.

It should be noted that all the observed variety of physical masses be forming of the particles of so-called electromagnetic block, i.e. by means of electric and magnetic spinor particles. Such masses can be called electromagnetic Masses. As for individual spinor particles as electric so and magnetic, they are all massless, so as (again) the mass is the result of their common structural organization. So, for example, the electron is massless spinor particles and no bosons including "divine" cannot change anything. Based on the results of his own experiments [1], [5] and the works of other researchers, the author came to a very unusual, from the perspective of modern physical conceptions the conclusion, that the atomic shells consisting of electric and magnetic spinor particles, are electromagnetic, rather than electronic, as it has always been considered. In this case, the number of magnetic spinor particles in atomic structures is approximately equal to the number of particles electric. It is the electromagnetic shells of the atoms are the natural sources (generators) of the gravitational field, which in reality is of the vortex electromagnetic field. Elementary source of the gravitational field is the electromagnetic spinor quasi-particle, which has received the name of its author s-graviton (s from the engl. source). In the composition of the s-graviton: two spinors (electron and magneton) and two corresponding antispinors. Figuratively, s-graviton can be represent in the form copula of electric and magnetic bispinors (bispinor this linked spinor pair: spinor and corresponding him antispinor) which revolve in antiphase to one atomic orbit. This quasiparticle can also be presented in the form of two joint orbital electric and magnetic currents, which revolves in antiphase. The above model of a coherent vortex electromagnetic current or s-graviton can be written in mathe- 
matical form as: $\operatorname{rot}\left[\boldsymbol{J}_{e}-\boldsymbol{J}_{g}\right]$, where $\boldsymbol{J}_{e}$ and $\boldsymbol{J}_{g}$-vectors of the instantaneous current density electric (e) and magnetic (g) charges corresponding to their vortex (circular) flows.

Then, the equation of the process of gravitational field formation by s-graviton can be presented in the form:

$$
\mathrm{k} \operatorname{rot}\left[\boldsymbol{J}_{e}-\boldsymbol{J}_{g}\right]=\operatorname{rot}[\boldsymbol{E}-\boldsymbol{H}]
$$

where $\boldsymbol{E}$ and $\boldsymbol{H}$ are vectors of instantaneous strength of electric and magnetic fields in the composition of the vortex electromagnetic (gravitational) field, $\mathrm{k}$ is the proportionality factor. Minus signs put in the above equation of the process gravitational field formation correspond both to reciprocal antiphase of orbital currents electrical and magnetic charges so and antiparallel orientation of vectors of instantaneous strength of electric and magnetic fields in every point of the gravitational field. As follows from the above Equation (1), the gravitational field is a vortex electromagnetic field, which is characterized by changes in the direction strength vectors $(\boldsymbol{E}$ and $\boldsymbol{H})$ electric and magnetic fields that are equal in magnitude and opposite in direction to each point. Unlike from vortex magnetic field, every point of that is answered by one vector of instantaneous strength of $\boldsymbol{H}$, every point of the elementary gravitational field is answered two vectors of instantaneous strength of the fields of $\boldsymbol{E}$ and $\boldsymbol{H}$ equal by value and oriented antiparallel each other. Vectorial-vortical analogy between magnetic (a) and gravitatinal (b) fields display in Figure 5.

In addition, Figure 5 shows that the vortex magnetic field is product of the revolving magnetic bispinor or, that too, of the revolving spinorial magnetic dipole. The source of the elementary gravitational field is the sgraviton that yet can defined as dynamic EM-dibispinor, i.e. two combined bispinors (electric and magnetic) circulating in antiphase on one the atomic orbitals. The classical equations of processes formation magnetic and electromagnetic (gravitational) vortex fields are also down on Figure 5. Gravitational fields corresponding vector condition: $<\operatorname{rot}[\boldsymbol{E}-\boldsymbol{H}]>\neq 0$, by analogy with the ferromagnetism can be called ferrogravitational fields (FGF) and the fields corresponding to the condition: $\langle\operatorname{rot}[\boldsymbol{E}-\boldsymbol{H}]>=0$, by analogy with the paramagnetism can be called paragravitational fields (PGF).

Between the masses (bodies) that emit paragravitational field is implemented a well-known attraction or gravity. The physical masses which emit ferrogravtational fields will push off from masses-sources a paragravitational fields, for example, from Earth, that constitute open by author present article Gravito-levitational effect (GLE) [16]. Below, in this article by author will show that the gravito-levitational effect is manifested such gases as hydrogen, $\mathrm{CO}_{2}, \mathrm{CH}, \mathrm{O}_{3}, \mathrm{CH}_{4}$, water vapor, which are called greenhouse gases. The physical cause, which determines the ability of the gases to flying or Gravito-levitation, is forming between molecules and earth positive "Dark Energy."

Note 4. Results of experiments author with magnetic charges and their currents in superconductors will allow him to develop technology to produce of the technical ferrogravitational field. Technical ferrogravitational

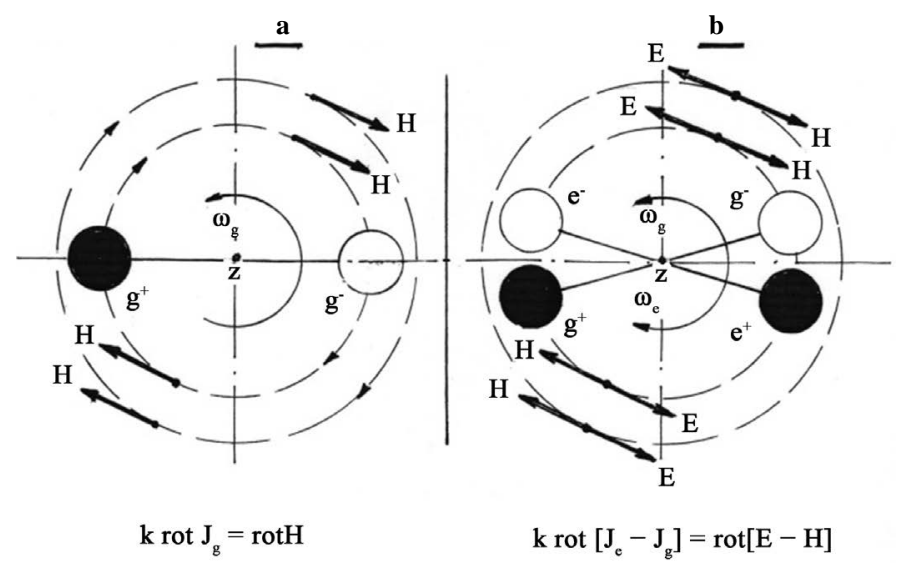

Figure 5. Schemes of mechanism the formation of the vortex fields: magnetic (a) and electromagnetic (gravitational) (b). White circles on the Figure 5 showed negative charged electric and magnetic spinors: electron $\left(\mathrm{e}^{-}\right)$and magneton $\left(\mathrm{g}^{-}\right)$and black circles-positively charged antispinors corresponding to them with charges $\mathrm{e}^{+}$and $\mathrm{g}^{+}$. 
fields and gravito-levitational forces formed by them can be used in transport, lifting and space engineering, power engineering, as well as in numerous gravitophysical and gravitochemical technologies (publications author's [7]-[9]).

It should be noted that the magnetic bispinors which inhabit on the outer orbitals in the shells of the so-called magnetic atoms (Fe, Co, $\mathrm{Ni}$ and others) are carriers of the so-called atomic magnetic moments and are responsible for all known magnetic phenomena in atoms.

In complete analogy with the process of formation of the vortex magnetic field is formed and the vortex electric field, which is described by the vortex vector rot $\boldsymbol{E}$. In this case, the source of such a field is a rotating electric bispinor with components: electric spinor, for example, an electron and the corresponding him real antimaterial particle-antispinor, which is the true antielectron. The main conclusion that follows from all stated above, is the author's assertion that the positive charges in the atoms and substance are actually real particles of Antimatter with their natural positive charge. It is important to emphasize that the electric antispinors, for example, antielectrons in substance are neither "holes" of Dirac, nor positrons. In Appendix II lists all the classical equations of electromagnetism with the inclusion of real magnetic charges, for the case of stationary (steady) processes, i.e. not time-dependent.

\section{Spinor Fields and "Dark Energy" in the Processes of the Climatic Changes on the Earth}

Let's note here an important conclusion of the author that all spinor fields, including the gravitational fields, are not a significant factor force action on the particles and the mass (of the body). The number energions, i.e. of particles Energo-medium, within the framework of spinor fields is less than $10^{9}$ their part, which lies in the volume of space in which there is a manifestation of the spinor field. A detailed analysis of the quantitative relationships energions in the composition of the spinor field, in relation to their total content in the volume Energo-medium, in which is implemented the impact this of the field, given in the books of the author [12] [13].

However spinor fields, due to the high density Energo-medium, able create in her composition a oblasts dissipation energion density, which in the form of active (power) zones, which referred to as "Dark Energy", has a direct physical impact (pressure) on the particles and the mass (of the body). In other words, the spinor fields able only play the role of "detonator" and under this yourself not have a significant power value. However, when exposed to such "detonators" on "explosives", role which takes the "Energo-medium", are implemented acts powerfully of force action of "Dark Energy" on particles of Matter and Antimatter, i.e. on substance, on the bodies and masses.

In order to estimate the ratio of the power capacity of spinor fields and "Dark Energy", which forming under their influence, it is useful to consider the process known in the technic as electro-hydraulic kick. This process is of the model analog of the mechanism of formation of "Dark Energy" and her force action on particles and bodies. The scheme of this process is shown in Figure 6, where shows a high-strength vessel 1, in the cover 2 which are mounted two electrodes 3 and 4 .

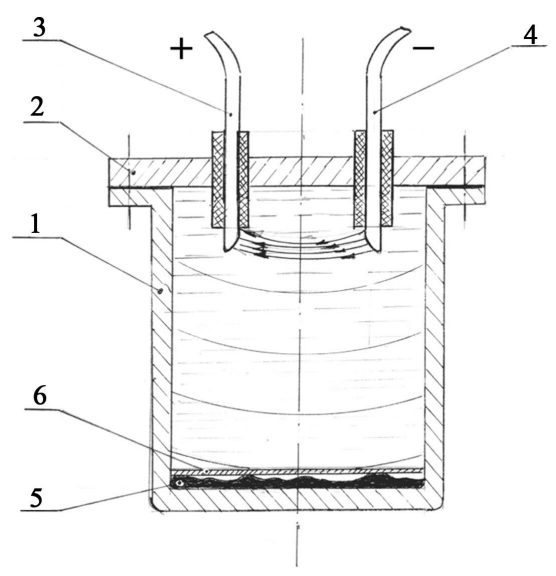

Figure 6. Electro-hydraulic effect as an illustration the model analogy of the process of formation and the impact of the forces of "Dark Energy". 
At the bottom of the vessel placed: matrix 5 and the metal blank 6 from sheet material. When implemented between the electrodes of a powerful electrical discharge in the vessel, which is completely filled with liquid, be created a high hydro-pressure (hydro-kick). As a result of the impact of hydro-kick on the metal blank she with great force is pressed against the matrix that allows you to make copies even of superhard materials. It is important to note that in the absence of liquid in the vessel, itself the discharge between the electrodes no significant effects on the metal blank has not. In the above model analogy, the electric arc plays the role of spinor field and the fluid that fills the vessel, performs the role of the Energo-medium, hydro-pressure in which acts in the role of "Dark Energy". Type or name of "Dark Energy" is determined by the nature of spinor fields, inducing her formation. For example, "Dark Energy", the formation of which is induced by static spinor fields, should be called as electrostatic or magnetostatic "Dark Energy". The formations of "Dark Energy" induced dynamic spinor fields, should be termed a dynamic "Dark Energy". The latter should include, for example, magneto-dynamic "Dark Energy", which is formed under the influence of vortex magnetic fields ( $\operatorname{rot} \boldsymbol{H})$, accompanying the passage of direct current of electric charges through a conductor. Dynamic can, apparently, be called "Dark Energy" induced by gravitational fields as ferrogravitational, so and paragravitational.

Another very important parameter of "Dark Energy" is this her sign. "Dark Energy" can be both positive and negative. Her sign depends on the sign of the gradient energo-pressure in oblast "Dark Energy" in relation to the average regional level of energo-pressure in Energo-medium. Thus, it is possible to define a positive "Dark energy" as a condition of $\frac{\mathrm{d} P}{\mathrm{~d} l}>0$, and negative as $\frac{\mathrm{d} P}{\mathrm{~d} l}<0$, where over the zero is adopted the average regional level of energo-density in Energo-medium. Therefore, different on nature and the structural organization of spinor fields induce in the composition of Energo-medium the "Dark Energy" of the various signs, reflecting the increase or decrease local levels of her energo-pressure.

On Figure 7 shows a force diagram corresponding to the scenarios: the formation of a negative "Dark Energy" between electric particles, which are charged charges of different signs (7a) and the formation of positive "Dark Energy" between particles with charges of the same sign ( $7 \mathbf{b})$. As noted above, these "Dark Energy" must be named as an electrostatic "Dark Energy".

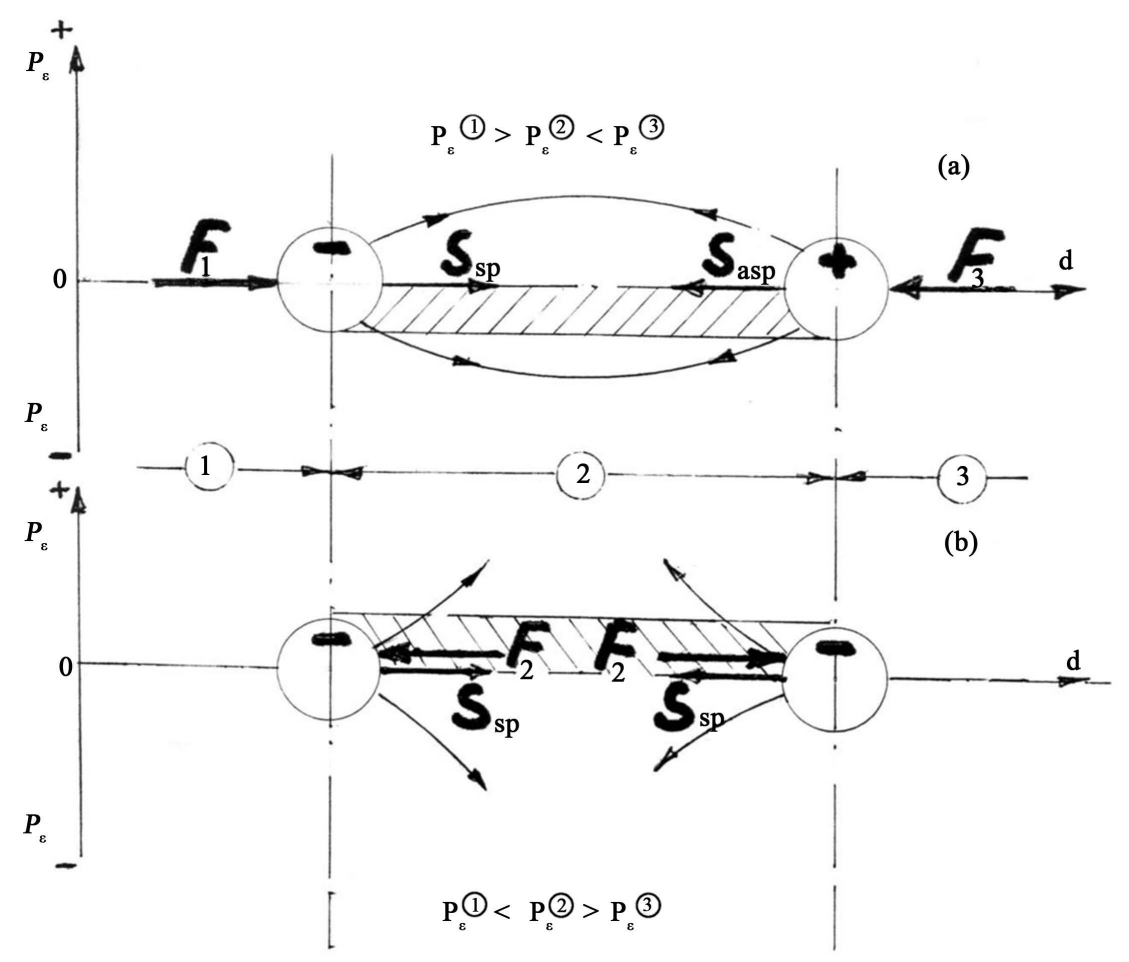

Figure 7. Power diagrams that corresponds to the formation: negative "Dark Energy" in the oblast between a pair of spinor particles with charges of different signs (a) and positive "Dark Energy" between a pair of the charged particles with charges of the same sign (b). 
In connection with the foregoing analysis of spinor fields and an introduction by the author the concept of positive and negative "Dark Energy", it is necessary to introduce concepts of open and closed of spinor fields, which are responsible different nature of impact on the energy state of Energo-medium. The open or mutually uncompensated spinor fields produce particles that are not located in such the spinor formations as bispinors.For example, spinor fields between two electrons (see Figure 7(b)), which create a zone of positive "Dark Energy" should be called as open spinor fields. Their mutual repulsion is proof that between the electrons is implemented positive "Dark Energy", the forces of which implements performing their repulsion. Closed or compensated spinor fields are fields that are realized in the framework of bispinors and called bispinors fields (see Figure 2). The closed spinor fields can be static, i.e. is forming by static bispinors and dynamic, formed a moving, more often by of rotating bispinors. For examples, of closed spinor fields are as gravitational fields so and vortex magnetic field which occur around conductors with a constant electric current. A distinguishing characteristic of the closed spinor fields are their ability to induce in the Energo-medium oblasts with reduced energo-pressure, i.e. the areas of negative "Dark Energy". In the area between the two so-called "paragravitational" atoms or between a pair of bodies are implemented the zone of negative "Dark Energy" (see Figure 8(b)). As a result of formation of such a zone is implemented the process of compressing (pressing) atoms or bodies to each other that in modern physical theory is defined as their mutual gravitation attraction. However, from the last process, there are very important exceptions. As noted in the previous section, between sources paragravitational and ferrogravitational fields are implemented discovered by the author gravito-levitational effect. The most powerful source paragravitational field is the Earth. Sources ferrogravitational field that participates in the implementation of gravito-levitational effect are, for example, so-called the greenhouse gases. Thus, between the molecules of greenhouse gases and of the Earth is implemented zones of positive "Dark Energy" which and contributes changes to the heat balance of our planet. Figure 8(a) shows the situation with the formation of the positive "Dark Energy" between a pair of sourses: one of which emits paragravitational field and the other field ferrogravitational. Source paragravitational field here is the Earth and as the source ferrogravitational field is the hydrogen atom. "Dark Energy", which is formed in this case, leads to repulsion of the sources of gravitational fields in a given pair. As noted above in the present article, this effect repulsion of the sources of paragravitational and ferrogravitational fields is the effect of Gravito-levitation.

Everything mentioned above concerning the formation and effects of "Dark Energy" on the particles and mass, may be associated with entropy as a measure of changes in regional and global Energo-medium. The last conclusion is particularly important from the point of view of the implementation of the possible regulation of the level of entropy (the level of energo-pressure) in the region of the Earth in connection with the problem of climatic changes. Technical human activities, usually accompanied by irreversible physical processes in the environment, which creates a higher concentration of positive "Dark Energy" and leads to an increase of entropy. It is interesting to note that greenhouse gases, such as $\mathrm{CO}_{2}, \mathrm{O}_{3}$ and $\mathrm{CH}_{4}$, are not themselves the main "guilty" of processes change the thermal balance on Earth. They add to this process a certain contribution, but it cannot, by itself, produce global climate change. However, these gases are correct indicator of the level of irreversible processes associated with the formation of so-called open spinor fields, which stimulating the growth of positive "Dark Energy", are the real culprits of climate change. It is easy to see that the greenhouse gases produce in

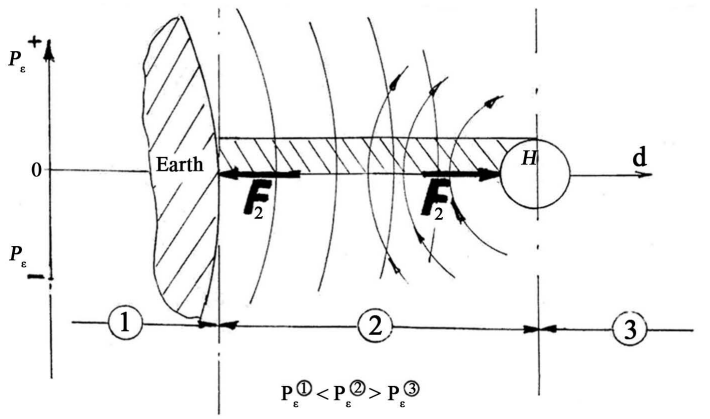

(a)

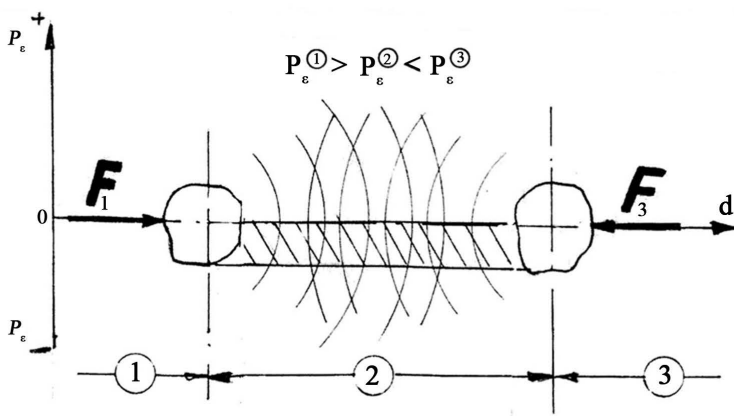

(b)

Figure 8. Schemes power reactions "Dark Energy", which is implemented in the following couple of the sources of gravitational fields: Earth (source paragravitational field) and a hydrogen atom (source ferrogravitational field) (a); two bodies, which emitting paragravitational field (b). 
technical processes such as: combustion, explosions, high-temperature reactions, in chemical technologies and many others.

Let us dwell on the generation of "Dark Energy" by the nuclear facilities. The fact that as a result of nuclear reactions, there is no "greenhouse" exit does not mean that these reactions are free from the "manufacture" positive "Dark Energy" and, hence, from growth her the thermal component in of the Earth's environment. In the various processes of atomic transformations that accompany nuclear reactions, formed high the density of the open spinor fields, which causes increase a positive "Dark Energy". As illustrate the last statement may be the observation of the bright glow of the atmosphere over the Chernobyl reactor, as in the time his of the explosion, so and minutes later. According to the author, this glow is a result impact of the positive of "Dark Energy" on air mass above the reactor. In addition to the ionization of the atoms of atmospheric gases, which determined the observed luminosity, was to take place increase the dynamic activity of air molecules, i.e., the corresponding temperature increase of the air mass. But for obvious reasons such measurements during the Chernobyl accident was not conducted.

Note in concluding this section that the author shows only some "outrageous" technologies that constantly improve the level of positive "Dark Energy" in the earth's environment. Extremely important is an extended analysis of those or other technical processes to identify their contribution to the formation of positive "Dark Energy”.

According to research by the author, all physical processes in the World who violate the order of spinor particles in the compositions bispinors and destroy the structure Masses (atoms, nucleons, substance, etc.), accompanied by the formation of a positive "Dark Energy". Conversely, the processes leading to the formation of bispinors and any the varieties of Mass, accompanied by the generation of a negative "Dark Energy.”

In addition, it is important to note that the "greenhouse gases" are involved in climate change on the Earth through education gravito-positive "Dark Energy”, which is a clear manifestation of Gravito-levitational effect.

\section{Conclusions}

Revealing magnetic spinor particles (magnetic charges) as real structural components of atoms and substance, as well as the determination of their key physical parameters, the author regards as the main result of his many years of experimental and theoretical studies (from 1969 to the present). The mechanisms of the processes of formation of magnetic field around a conductor with a constant electric current, the physics of the Gravitational field, the structural organization of Physical Mass, an electromagnetic device shells of atoms and other physical conditions and manifestations been cleared up on the basis of the above main results by means of simple physical logic and modeling. Magnetic spinor particles and their fields, that missing link in the system of the basic physical presentations, without which it is impossible to understand the physical picture of the real World. As noted above, the main reasons which detained on 140 years official recognition of real magnetic charges and related with them important technical applications, were as fundamental differences in the Physics of retention (confinement) of magnetic spinor particles and electrons in atoms and the substance, so and the vicious concept of electromagnetism J. C. Maxwell [17]. In the framework of this concept, Maxwell, in the absence of knowledge about the real physical processes occurring in the conductor with electric current, arbitrary decision deprived magnetic field of his own source, i.e. of the magnetic pole (magnetic charge). Largely, because of a fallacious electromagnetic Maxwell's concept, magnetic charges were "buried alive" and the magnetic field that they emit, have transformed into theoretical "miscarriage" of the electricity. Besides, "a cruel joke" played the Physics of confinement of the spinor particles in substance, for example, with positive electric charges (with real Antielectrons), which with the filing by P. Dirac [18], was mistakenly recognized as "holes” or electronic vacancies.

The research of the real magnetic spinor particles in the atoms and substances and the elucidation of the natural status of the so-called electron vacancies or "holes", enabled author to strengthen in the idea, that the atomic shells are electromagnetic, i.e. consist of electric and magnetic spinor particles. The next important step was the formation by author of the concept of Physical Triad of real World. Using the concept of the Physical Triad, the author was able to get clear answers on questions, such as those associated with Physics of real spinor particles Matter and Antimatter and also with mechanisms of processes of formation of spinor fields in the Energo-medium. Very important is the conclusion of the author about the existence of Antimatter as real and an independent component of the Physical Triad. Antimatter and Antisubstance are completely different physical catego- 
ries. "Absence" Antimatter and its particles (antispinors) in physical representations, in the same way as the "absence" of the magnetic spinor particles, are caused, first of all, by the Physics of confinement spinor particles in atoms and substance.

As a certain example of application of the physics worked out by an author and expounded in this article, is demonstration role of positive "Dark Energy" which by caused technical activity of man, in the processes of change of climate on Earth. The author considers the mechanisms of the processes of formation of "Dark Energy" and its role in the global force effects on the particles and the mass as in a cosmic scale and in the microcosm. Real physical factors, which are caused by technical human activity and have a direct impact on the thermal regime of the Earth, are open spinor fields and positive "Dark Energy", which is induced by such fields. With regard to greenhouse gas emissions, they are only one of a number of factors, which pumps up Energo-medium of positive "Dark Energy." Under this, greenhouse gases are a pretty good indicator of the overall situation from a technical heat flows that determine the negative changes the Earth's climate.

Note 5. The main provisions of General Physics with real magnetic charges were reported at the International PIERS 2009 Moscow, conference (Progress in Electromagnetics Research Symposium [19] [20]).

\section{Acknowledgements}

The author is Grateful to technical specialists: Davydov A.A., Koshelev N.V., Lomakin A.I., Moiseenkov E.V. and Staritsin S.E., which provide technical support for the main part of his experiments, concerned the researches of magnetic charges and Physics of gravitational field.

\section{References}

[1] Sizov, R.A. (2001) New Presentation of Nature Magnetism, Gravitation and Nuclear Forces of Bonding (Experimentsand Theory). Akademizdat Center "Science", Moscow.

[2] Sizov, R.A. (1970) Solid State Physics, 12, 2869-2875.

[3] Sizov, R.A. (1971) Journal of Experimental and Theoretical Physics, 60, 1363-1370.

[4] Sizov, R.A. (2005) Magnetic Elementary Particles as Stable Structural Components of Atoms and Substance (Experimentsand Theory). Akademizdat Center "Science", Moscow.

[5] Sizov, R.A. (2008) Electric and Magnetic Spinorial Particles as Structure-Forming Components of Mass and Electromagnetic Source Gravitation (Experiments and Theory). Akademizdat Center "Science", Moscow.

[6] Sizov, R.A. (2015) Journal of Modern Physics, 6, 1013-1022. http://dx.doi.org/10.4236/jmp.2015.68106

[7] Sizov, R.A. (2013) Transference of People and Loads in the Terrestrial Space by Means of Technical Ferrogravitation. Akademizdat Center "Science”, Moscow.

[8] Sizov, R.A. (2013) Generation and Technical Application of Ferrogravitation (The Collection of the Invention of theAuthor in Area the Gravitophysical and Gravitochemical Technologies). Akademizdat Center "Science”, Moscow.

[9] Sizov, R.A. (2015) Journal of Modern Physics, 6, 1591-1601. http://dx.doi.org/10.4236/jmp.2015.611161

[10] Ehrenhaft, F. (1910) Wiener Berichte, 119, 836.

[11] Ehrenhaft, F.(1942) Journal of the Franklin Institute, 233, 235-256. http://dx.doi.org/10.1016/S0016-0032(42)90311-9

[12] Sizov, R.A. (2015) Journal of Modern Physics, 6, 2280-2289. http://dx.doi.org/10.4236/jmp.2015.615232

[13] Sizov, R.A. (2011/2012) Matter, Antimatter and Energo-Medium-Physical Triad of the Real World. AkademizdatCenter "Science", Moscow.

[14] Atsukovsky, V.A. (2001) Physical Principles of Electromagnetism and Electromagnetic Phenomena. Ether-Dynamic Interpretation, Moscow.

[15] Le Sage, G.-L (1784) Annual Report of the Board of Regents of the Berlin, 404-432.

[16] Sizov, R.A. (2012) Levitation as a Spread Force Manifestation of Natural Ferrogravitation. Akademizdat Center "Science", Moscow.

[17] Maxwell, J.C. (1873) Treatise on Electricity and Magnetism. Volume 1-2, Clarendon Press, Oxford.

[18] Dirac, P.A.M. (1930) Proceedings of the Royal Society A, 126, 360. http://dx.doi.org/10.1098/rspa.1930.0013

[19] Sizov, R.A. (2009) Magnetic Particles (Magnetons)—Structural Components of Atoms and Substance, ImmediateSources of Magnetic Field. Theory and Experiments, the Report at PIERS 2009, Moscow.

[20] Sizov, R.A. (2009) Electric and Magnetic Spinor Particles-The Electromagnetic Source of Gravitation. Theory and Experiments, The Report at PIERS 2009, Moscow. 


\section{Appendix I}

The main physical parameters of the magnetic and electric spinor particles (magnetons, antimagnetons and antielectrons) constituting, together with electrons of atomic shell.

Charge: The magneton (magnetic spinor) and the antimgneton (magnetic antispinor) have a magnetic charges $g$ the value of which is equal to the value of the electron charge ( $g=e)$. Researches of the author showed that the greatest possibility charge of a fundamental magnetic spinor particle is equal to electron charge, i.e. $g_{\max }=$ e.

Sign of the charges: Like an electron a magneton has a charged with the sign "minus" (g”). Antimagneton has a positive magnetic charge $\left(\mathrm{g}^{+}\right)$.

Mass: Like an electron a magneton,antimagneton and antielectron are a massless particles, because the physical mass is result of joint structural "activity" of electric and magnetic spinor particles.

Class of elementary particles: The magneton is a lepton and the antimagneton and antielectron-an antileptons.

Statistical properties: The magneton is a fermion (spin equal $1 / 2$ ) and the antimagneton and antielectron-an antifermions (spin equal $-1 / 2$ ).

The relation to phases of the Physical Triad: the Electron and Magneton are the material particles i.e. they are particles the Material Phases of Physical Triad. The antielectron and antimagneton are the particles of Antimatter.

\section{Appendix II}

The classic equations of electromagnetic states whis electric and magnetic charges.

$$
\begin{aligned}
& \text { 1. } \operatorname{div} \mathrm{E}=4 \pi \rho_{\mathrm{e}} \\
& \text { 2. } \operatorname{div} \mathrm{H}=4 \pi \rho_{\mathrm{g}} \\
& \text { 3. } \operatorname{rot} \mathrm{E}=0 \\
& \text { 4. } \operatorname{rot} \mathrm{H}=0 \\
& \text { 5. } \operatorname{div} \mathrm{H}^{\circ}=0 \\
& \text { 6. } \operatorname{div} \mathrm{E}^{\circ}=0 \\
& \text { 7. } \mathrm{k}_{1} \mathbf{J}_{\mathrm{e}}=\operatorname{rot} \mathbf{J}_{\mathrm{g}}^{\circ} \\
& \text { 8. } \mathrm{k}_{1} \mathbf{J}_{\mathrm{g}}=\operatorname{rot} \mathbf{J}_{\mathrm{e}}^{\mathrm{o}} \\
& \text { 9. } \mathrm{k}_{2} \operatorname{rot} \mathbf{J}_{\mathrm{g}}^{\mathrm{o}}=\operatorname{rot} \mathrm{H}^{\circ} \\
& \text { 10. } \mathrm{k}_{2} \operatorname{rot} \mathbf{J}_{\mathrm{e}}^{\mathrm{o}}=\operatorname{rot} \mathrm{E}^{\circ} \\
& \text { 11. } \mathrm{k}_{1}\left(\mathbf{J}_{\mathrm{e}}+\mathbf{J}_{\mathrm{g}}\right)=\operatorname{rot}\left[\mathbf{J}_{\mathrm{e}}^{\circ}-\mathbf{J}_{\mathrm{g}}^{\circ}\right] \\
& \text { 12. } \mathrm{k}_{2}\left(\mathbf{J}_{\mathrm{e}}^{\mathrm{o}}+\mathbf{J}_{\mathrm{g}}^{\mathrm{o}}\right)=\operatorname{rot}\left[\mathrm{E}^{\mathrm{o}}-\mathrm{H}^{\circ}\right]
\end{aligned}
$$

$$
\text { Designation }
$$

E, $\mathrm{H}$ - vectors strength of

electrostatic and magnetostatic fields,

$\rho_{\mathrm{e}}, \rho_{\mathrm{g}}$ - density of the electric (e)

and magnetic $(\mathrm{g})$ charges,

$\mathrm{E}^{\mathrm{o}}, \mathrm{H}^{\circ}$ - vectors instantaneous strength of the vortical of electric and magnetic fields,

$\mathbf{J}_{\mathrm{e}}^{\mathrm{o}}, \mathbf{J}_{\mathrm{g}}^{\mathrm{o}}$ - vectors instantaneous

density of the vortical currents electric and magnetic charges,

$\mathrm{J}_{\mathrm{e}}, \mathrm{J}_{\mathrm{g}}$ - vectors density of linear currents electric and magnetic charges,

$\mathrm{k}_{1}, \mathrm{k}_{2}$ - the coefficients of

proportionality 